\title{
Laser-Induced Fluorescence of Wet Porous Silicon as Laser-Induced Fluorescence of $\mathrm{H}_{3} \mathrm{O}^{+}$
}

\author{
Yuri Pivovarenko \\ Research and Training Centre 'Physical and Chemical Materials Science' Under Kyiv Taras Shevchenko University and NAS of Ukraine, \\ Kiev, Ukraine
}

Email address:

y.pivovarenko@gmail.com

To cite this article:

Yuri Pivovarenko. Laser-Induced Fluorescence of Wet Porous Silicon as Laser-Induced Fluorescence of $\mathrm{H}_{3} \mathrm{O}^{+}$. Journal of Photonic Materials and Technology. Vol. 5, No. 1, 2019, pp. 11-15. doi: 10.11648/j.jmpt.20190501.13

Received: March 7, 2019; Accepted: April 12, 2019; Published: May 20, 2019

\begin{abstract}
Typically, the production of porous silicon is an electrochemical etching of monocrystalline silicon wafers, which are connected to the anode, in ethanol-aqueous HF solutions. In the process of etching, it turns out porous silicon, which is saturated with water, which is rich in protonated molecules $\mathrm{H}_{3} \mathrm{O}^{+}$, formed as a result of a number of well-known physicochemical processes. At the same time, the presence of molecules of such protonated water in the composition of freshly prepared porous silicon is usually ignored. At the same time, both the ability of protonated water to fluoresce under the action of laser radiation in the UV range, and the possible contribution of such fluorescence to the total fluorescence of porous silicon induced by a UV laser is ignored. Since such ignoring seems to be incorrect, the possible contribution of laser fluorescence of water enriched with its protonated molecules to the laser fluorescence of moistened porous silicon is discussed here. Since this may be important for the correct interpretation of the results obtained when studying the spectra of laser-induced fluorescence of porous silicon moistened with aqueous solutions, in particular - with aqueous solutions of biological substances, the unique properties of such water are also demonstrated. Thus, the exceptional penetrating ability of positively charged water is visualized, due to which it is able to transfer from the hydrated shells of biopolymers to porous silicon and enhance its laserinduced fluorescence. It also demonstrates the exceptional ability of positively charged water to evaporate; which makes it possible to explain the rapid disappearance of the fluorescence of porous silicon, which is observed during its drying.
\end{abstract}

Keywords: Fluorescence, Porous Silicon, Positively Charged Water

\section{Introduction}

Due to its laser-induced fluorescence in the visible region, porous silicon is considered as a promising material for the development of various sensors. Traditionally, the production of porous silicon consists of electrochemical etching of single-crystal silicon wafers, which are connected to the anode, in ethanol-aqueous solutions of HF. Despite the fact that the method of preparation of porous silicon remains almost unchanged for many years, its ability to fluoresce is still not fully understood [1-4]. Given this, we sought to attract the attention of researchers to certain processes that necessarily occur during the preparation of porous silicon.

So, the preparation of porous silicon is the processing of monocrystalline silicon with aqueous solutions of HF [1-4], which are enriched with protonated water molecules $\left(\mathrm{H}_{3} \mathrm{O}^{+}\right)$ resulting from the following reaction: $\mathrm{H}_{2} \mathrm{O}+2 \mathrm{HF} \leftrightarrow \mathrm{H}_{3} \mathrm{O}^{+}+$
$\mathrm{HF}_{2}^{-}$[5]. In addition, protonated water molecules appear in the composition of the plates of anodized monocrystalline silicon [1-4] as a result of the addition of protons, which are formed in the anode section during electrolysis: $2 \mathrm{H}_{2} \mathrm{O}-4 \mathrm{e}^{-}$ $\rightarrow \mathrm{O}_{2}{ }^{\uparrow}+4 \mathrm{H}^{+}$[5]. Finally, protonated water molecules can form in water, which is in contact with oxygen gas bubbles [5] released at the anode. Thus, in the process of preparing porous silicon, several well-understood processes take place, thanks to which it is enriched with protonated water molecules.

All these processes are of interest, since the vapor, which is enriched with protonated water molecules $\mathrm{H}_{2} \mathrm{O}^{+}$, has a distinct laser-induced fluorescence in the $400-750 \mathrm{~nm}$ wave region with a sharp maximum at $668.6 \mathrm{~nm}$ (Figure 1) [6].

At the same time, the unconditional presence of a finely dispersed positively charged water layer in freshly prepared porous silicon and its ability to laser-induced fluorescence are not taken into account when explaining the laser-induced 
fluorescence of porous silicon [1-4].

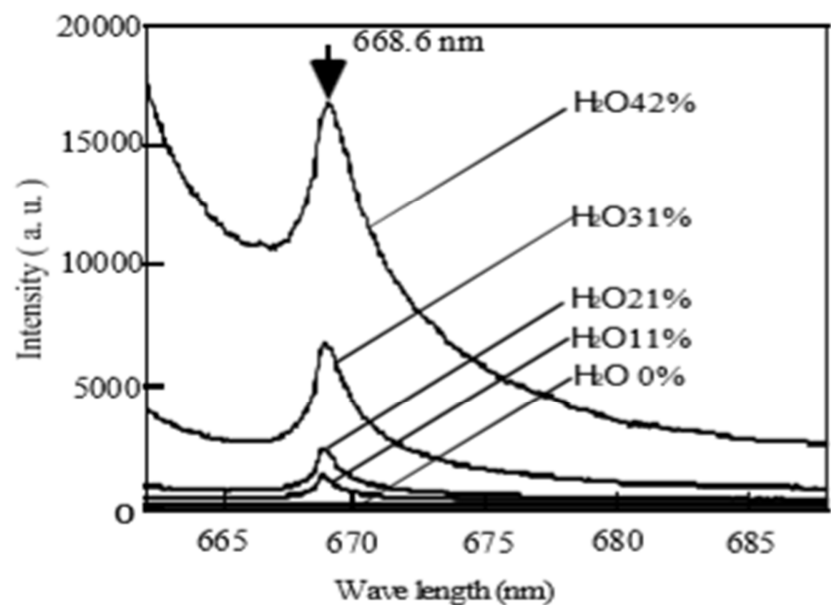

Figure 1. The spectrum of laser-induced fluorescence of a positively charged vapor [6].

Since this seems necessary, we are discussing here the possible contribution of laser-induced fluorescence of positively charged water to laser-induced fluorescence of moistened porous silicon. Also discussed here are the unique properties of such water.

\section{Main Body}

\subsection{Laser-induced Fluorescence of Positively Charged Water}

The estimated presence of positively charged water in porous silicon is confirmed by some of the raw spectra of laser-induced fluorescence of freshly prepared porous silicon, on which peaks appear at $668.6 \mathrm{~nm}$ (Figure 2, 1-day spectrum), corresponding to the maximum laser-induced fluorescence of positively charged water vapor [6].

Moreover, all presented spectra (Figure 1) confirm the sorption of positively charged water by freshly prepared porous silicon. The last statement becomes clear if we consider that only (!) positively charged water can evaporate $[7,8]$. Thus, the drying of freshly prepared porous silicon, like any drying, consists in reducing the content of exclusively positively charged water, the loss of which by porous silicon reflects the time evolution of its laser-induced fluorescence, apparently (Figure 2).

Thus, freshly prepared porous silicon retains the ability to fluoresce until the positively charged water that it has absorbed during its preparation evaporates.

It should be noted separately that positively charged water has some truly unique properties that deserve special attention, since they are important for correct interpretation of the results obtained in the study of the spectra of laserinduced fluorescence of porous silicon moistened with aqueous solutions of biological substances. First of all, such properties should include the extremely high penetrating force of positively charged water, which, in particular, determines its pronounced ability to hydrate various substances, in particular, biological polymers, and therefore to form their hydrated shells.

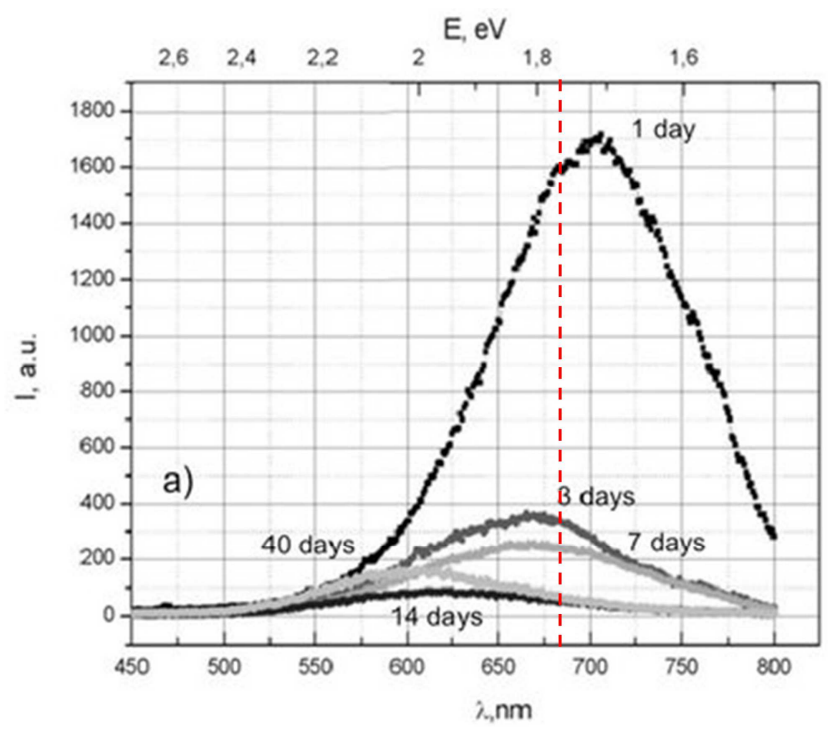

Figure 2. The spectrum of laser-induced fluorescence of freshly prepared porous silicon (1 day) and its evolution over time. Spectra were not processed.

The red dotted line represents $668.6 \mathrm{~nm}$, which corresponds to the maximum laser-induced fluorescence of a positively charged vapor [6].

\subsection{High Hydrating and Penetrating Abilities of Positively Charged Water}

It is easiest to verify the high penetrating power of positively charged water by observing the swelling of starch in uncharged water, as well as in negatively or positively charged water. So, you can make sure that starch never swells in uncharged or negatively charged water at room temperature (Figure 3, left), and in positively charged water at room temperature it swells within a few minutes (Figure 3, right) [9-11].

Since a similar ability of positively charged water was observed with respect to other biopolymers, including DNA [11], it can be concluded that hydrated shells of swollen biopolymers are formed mainly from positively charged water. (It is also useful to take into account that it is precisely the positively charged water that hydrates glucose, providing positive electrization of its molecules in aqueous solutions [5].)

The uniqueness of positively charged water is not limited to this. So, it can be seen that positively charged water evaporates even from closed plastic bottles (Figure 3, right) $[9,10]$, which demonstrates its exceptional penetrating ability. Given this, it is not surprising that positively charged water can easily penetrate into the pores of porous silicon. It is also natural to assume that the sizes of particles of positively charged water, which penetrate into plastic and, presumably, into the pores of porous silicon, are as small as particles of vapor; taking into account the technology of preparation of porous silicon [1-4], it is important to note that 
solutions that form in the anode compartments of electrolyzers also have a similar penetrating power.

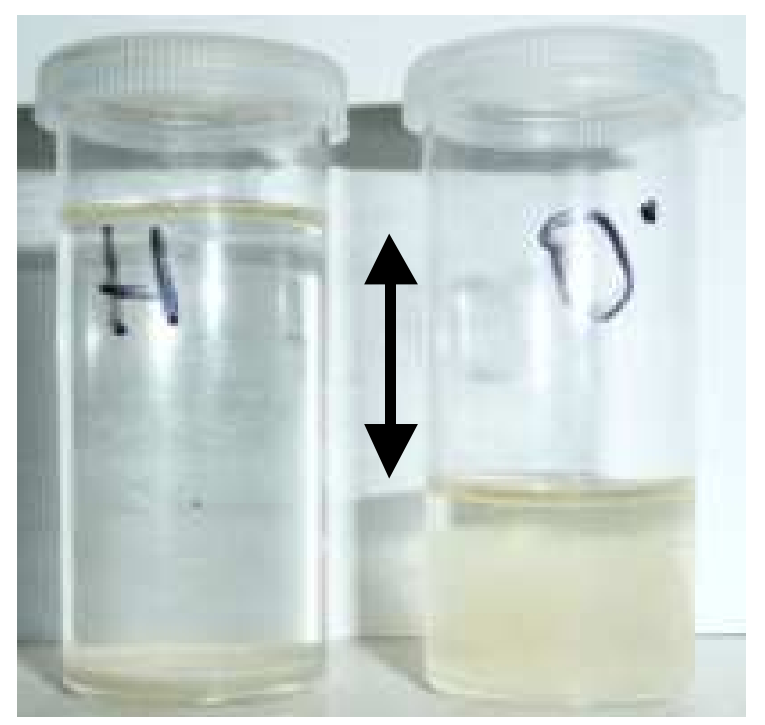

Figure 3. There is a swelling of starch in water $\left(\sim 20^{\circ} \mathrm{C}\right)$ with a different electric potential. Starch for months does not swell in water with a potential of $-250 \mathrm{mV}$ (left), but swells in water with a potential of $+250 \mathrm{mV}$ in a few minutes (right). The electric potentials of electrified waters were measured relative to uncharged water.

Water with negative potential was obtained by bubbling uncharged water with hydrogen gas (left); water with a positive potential was obtained by bubbling uncharged water with gaseous oxygen (right). The same results were obtained when using solutions from the cathode and anode sections of the electrolyzer.

Water with a positive potential can evaporate even from a closed plastic flagon: the arrow shows how much during the day the level of such water has decreased [9-11].

A similar pattern is also observed when the DNA swells [11].

It should be noted that similar penetrating power also appears in water irradiated with ionizing radiation [12].

\subsection{The Nature of Laser-Induced Fluorescence of Porous Silicon Moistened (Modified) with Aqueous Solutions of Polymers of Biological Origin}

Thus, it can be assumed that the laser-induced fluorescence of porous silicon modified with aqueous solutions of biopolymers is due, in fact, to the fluorescent particles of positively charged water, which pass from the hydrated shells of biopolymers to the pores of porous silicon. Given this, we can conclude that laserinduced fluorescence of porous silicon modified with aqueous nucleic acids reflects, in fact, their hydration. For this reason, we came to the conclusion that our previously obtained spectra (Figure 4, spectra 2, 3) reflect not different oxidation of DNA and RNA with active oxygen, as we assumed [13], but a wellknown fact: DNA molecules are more hydrated than RNA molecules [14].

In addition, changes in the intensity of laser-induced fluorescence of porous silicon moistened with aqueous solutions containing the enzymes urease and glucose oxidase and their substrates [4] should be interpreted taking into account the demonstrated difference (Figure 2). Thus, an increase in the intensity of laser-induced fluorescence of porous silicon, moistened with aqueous solutions that contain glucose oxidase and increasing concentrations of $\mathrm{H}_{2} \mathrm{O}_{2}$ [4], reflects an increase in the humidity of porous silicon due to an increase in the concentration of atomic oxygen $\mathrm{O}^{*}: \mathrm{H}_{2} \mathrm{O}_{2}$ $\rightarrow \mathrm{H}_{2} \mathrm{O}+\mathrm{O}^{*}$ [5]. Accordingly, a decrease in the intensity of laser-induced fluorescence of porous silicon moistened with aqueous solutions that contain urease and increasing concentrations of its substrates [4]reflects a decrease in water content in porous silicon due to an increase in atomic hydrogen concentration $\mathrm{H}^{*}: 4 \mathrm{H}_{2} \mathrm{CO}_{3} \rightarrow 2 \mathrm{H}_{2} \mathrm{O}+4 \mathrm{CO}_{2}+4 \mathrm{H}^{*}$ [5]. Thus, taking into account the demonstrated difference (Figure 2), the changes [4] registered by the authors actually reflect the hydration or dehydration of porous silicon.

A.U.

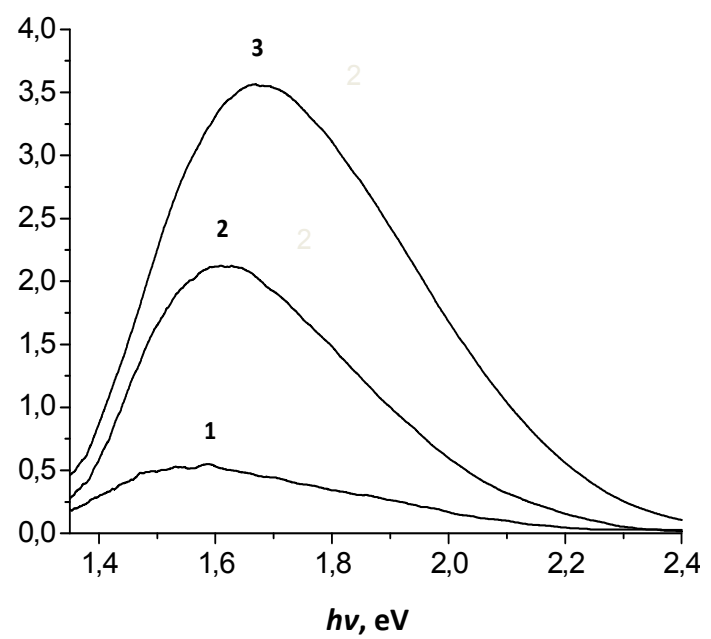

Figure 4. Laser-induced fluorescence spectra of porous silicon: 1 unmodified porous silicon; 2 - porous silicon modified with an aqueous solution of RNA; 3 - porous silicon modified with an aqueous solution of $D N A$.

Modification of porous silicon consisted in its wetting with an aqueous solution of RNA and DNA and subsequent drying under room conditions. [13].

The wavelength of the exciting laser radiation was $337 \mathrm{~nm}$

\subsection{Shifts in the Spectra of Laser-Induced Fluorescence of Moistened Porous Silicon}

Previously obtained results suggested that gaseous hydrogen can fluoresce in aqueous media, forming peaks at wavelengths that correspond to Ballmer series lines [15]. Testing the ability of atomic hydrogen to fluoresce in aqueous media consisted in the study of the fluorescence of water that came in contact with various sources of atomic hydrogen. In particular, the fluorescence of water in contact with iron powder was studied, - in this case, hydrogen atoms are formed during the reaction between metallic iron and water: $\mathrm{Fe}+\mathrm{H}_{2} \mathrm{O} \rightarrow \mathrm{Fe}(\mathrm{OH})_{2}+2 \mathrm{H}^{*}$ [5]. As a result of 
suchexperiments, the occurrence of fluorescence with a maximum at $423 \mathrm{~nm}$ was recorded (Figure 5, the right spectrum).

Obviously, this peak can be represented as the sum of two peaks with maxima 410.17 and $434.04 \mathrm{~nm}$, which correspond well to the two lines of the Ballmer series: $410.17(\mathrm{n}=6$, violet line $)+434.04(\mathrm{n}=5$, blue line $) / 2=773.4 / 2=$ $422.105 \mathrm{~nm}$ [16]. When studying the fluorescence of water contacting metallic iron, it was found that the initial peak with a maximum at a wavelength of $423 \mathrm{~nm}$, eventually shifted to short-wave region up to $364.56 \mathrm{~nm}$, which corresponds to the shortwave boundary of the Ballmer series $(\mathrm{n}=\infty)$ (Figure 5, the left spectrum) [16]. The reasons for this shift are still not clear, but this phenomenon has been recorded many times.

\section{A.U.}

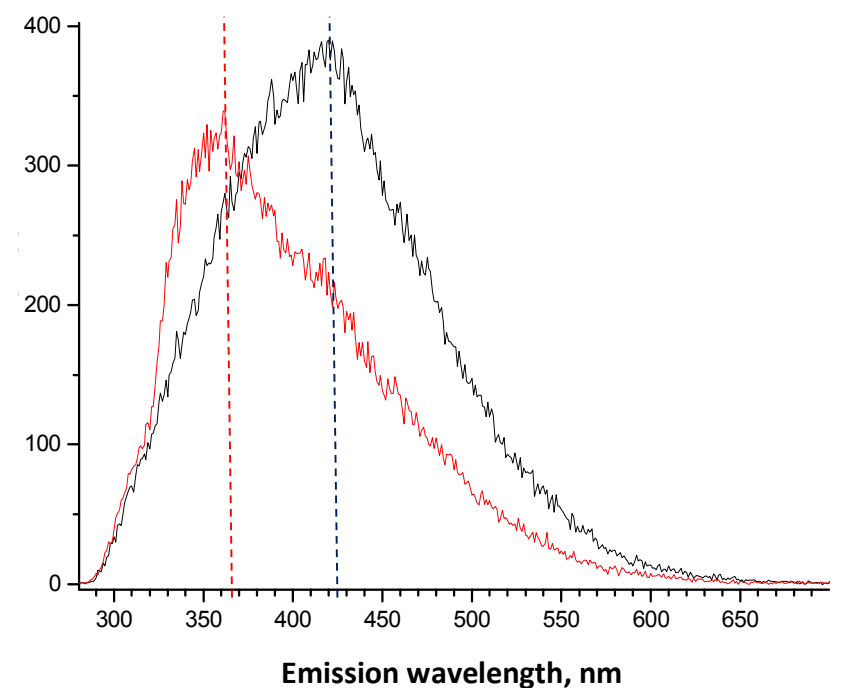

Figure 5. These are the fluorescence spectra of water reacted with metallic iron: $\mathrm{Fe}+\mathrm{H} 2 \mathrm{O} \rightarrow \mathrm{Fe}(\mathrm{OH}) 2+2 \mathrm{H}^{*}$. The black curve is a spectrum recorded 30 minutes after the start of the reaction; the red curve is a spectrum recorded 10 hours after the end of the reaction.

The blue dotted line shows a wavelength of $\sim 423 \mathrm{~nm}$ resulting from the summation of the two Ballmer series lines, $410.17 \mathrm{~nm}$ (violet line) and $434.04 \mathrm{~nm}$ (blue line): $(410.17+$ 434.04) / $2=773.4 / 2=422.105 \mathrm{~nm}$; it corresponds to a lower activity of aqueous hydrogen atoms. The red dotted line shows the shortwave boundary of the Ballmer series (364.56 nm).

The excitation wavelength of the fluorescence was 230 $\mathrm{nm}$. The working solution was at room temperature throughout the experiment.

Trying to explain the reasons for this shift, we assumed that it reflects the general distribution of atmospheric charges. So, it is known that positive atmospheric charges move upwards, and negative ones - downwards [7, 8]; the polarization of clouds convincingly demonstrates this distribution of atmospheric charges (Figure 6).

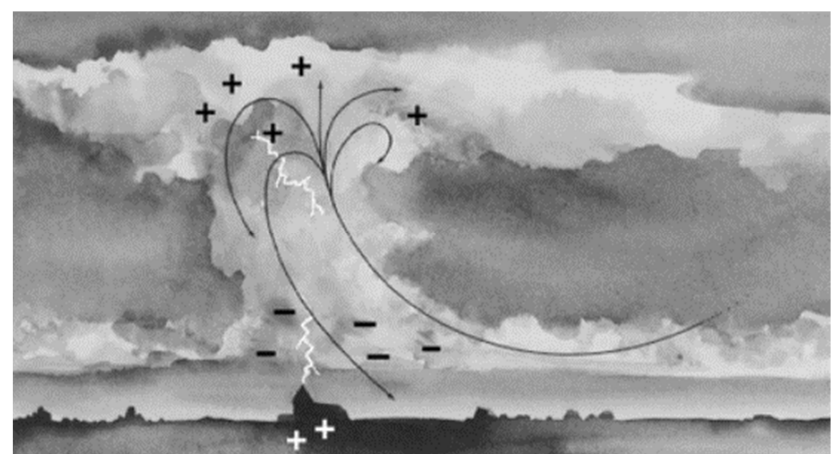

Figure 5. Polarization of clouds: the lower part of atypical cloud has a negative charge and the upper part has appositive charge. Positively charged water vapor is concentrated in the upper part of the clouds, and negatively charged the lower part [7,8].

Thus, the stored aqueous solutions are more and more enriched with negative charges, like the lower parts of clouds (Figure 6), which can lead not only to the formation of hydrogen atoms (in general, so: $\mathrm{e}^{-}+\mathrm{H}^{+} \rightarrow \mathrm{H}^{*}$ ), but also to an increase in their concentration. Given this, it can be assumed that the short-wave shift in the fluorescence spectra of aqueous solutions containing atomic hydrogen (Figure 4) reflects an increase in its concentration. So, it must be remembered that most experiments are conducted under terrestrial conditions, i.e. - on the surface of a rotating Earth and under the influence of the geomagnetic field.

Let us return to the shift of the spectra of laser-induced fluorescence of moistened porous silicon (Figure 2). It is known that the position of the maxima of the spectra of such fluorescence depends on the size of the nanoparticles (porosity) of porosity silicon. So, it was shown that a maximum of $690 \mathrm{~nm}$ corresponds to particles with a size of $3.2 \mathrm{~nm}$, and a maximum of $390 \mathrm{~nm}$ corresponds to particles with a size of $1.5 \mathrm{~nm}$ [4]. Taking this into account, we can conclude that the location of such maxima is determined by the ability of porous silicon to absorb water, first of all, water enriched with its protonated molecules $\left(\mathrm{H}_{3} \mathrm{O}^{+}\right)$.

Since the positive electrification of water is accompanied by an increase in its surface tension [9, 10], it can be assumed that a strong film with an increased concentration of protonated molecules $\left(\mathrm{H}_{3} \mathrm{O}^{+}\right)$can form on the surface of porous silicon. Thus, the short-wavelength shift of the spectra of laser-induced fluorescence of porous silicon can reflect an increase in the density of protonated water molecules $\left(\mathrm{H}_{3} \mathrm{O}^{+}\right)$ in the film covering its surface.

Analyzing the possibility of the formation of such a water film, one should keep in mind the rule of Kyon: upon contact of the two phases, the phase which has high dielectric permittivity receives a positive charge [5]. Since the dielectric constant of water at room temperature is $\sim 81$ [5], and the dielectric constant of porous silicon is one to two orders of magnitude less [17], the water film that contacts the porous silicon certainly acquires a positive charge. Thus, in addition to the above-described reasons for positive charging of the aqueous layer covering porous silicon, there is an additional reason, which is realized with close contact of water and porous silicon. 
Thus, both discussed types of short-wavelength shifts of fluorescence maxima can be caused by an increase in the concentration of fluorogen. In any case, the short-wavelength shift of the fluorescence maxima does not belong to exceptional phenomena.

\section{Conclusion}

The laser-induced fluorescence of moistened porous silicon can be, at least in part, the laser-induced fluorescence of water enriched with its protonated molecules $\left(\mathrm{H}_{3} \mathrm{O}^{+}\right)$, which is integrated into the pores of porous silicon. This view is very productive. So, given that the hydrate shells of biopolymers consist mainly of positively charged water, it can be assumed that laser-induced fluorescence of porous silicon, moistened with aqueous biopolymers, reflects the degree of their hydration. Moreover, given that the conformation of biopolymers, and therefore their activity, is sensitive to their hydration, it can be concluded that laserinduced fluorescence of porous silicon, moistened with aqueous biopolymers, may indirectly correlate with both the degree of their hydration and their biological activity.

\section{References}

[1] Feng Z. and Tsu R. (1994) Porous Silicon. Singapore: World Scientific. ISBN 981-02-1634-1633

[2] Zhou Z., Brus L. and Friesner R. (2003) Electronic Structure and Luminescence of 1.1- and 1.4-nm Silicon Nanocrystals: Oxide Shell versus Hydrogen Passivation, Nano Lett, 3, 163 167

[3] Sailor M. J. (2012) Porous Silicon in Practice: Preparation, Characterization and Applications. Weinheim: Wiley-VCH

[4] Syshchyk O., Skryshevsky V. A., Soldatkin O. O. and Soldatkin A. P. (2015) Enzyme biosensor systems based on porous silicon photoluminescence for detection of glucose, urea and heavy metals // Biosensors and Bioelectronics, 66, 89-94
[5] Nekrasov B. V. (1974) Bases general chemistry, 1. Moscow: Chemistry. In Russian

[6] Lew H. (1976) Electronic spectrum of $\mathrm{H}_{2} \mathrm{O}^{+}$. Canadian Journal of Physics, 54(20), 2028-2049

[7] Krasnogorskaya N. V. (1984) Electromagnetic fields in the atmosphere of the Earth and their biological significance, 1. Moscow: Nauka. In Russian

[8] Pivovarenko Y. (2015) A Charge Distribution in the Earth's Atmosphere. American Journal of Physics and Applications, 3(3), 67-68

[9] Pivovarenko Y. (2017) Potential-Dependent Changes of the Surface Tension of Water. Fluid Mechanics, 3(4), 29-32

[10] Pivovarenko Y. (2018) \pm Water: Demonstration of water properties, depending on its electrical potential. World Journal of Applied Physics, 3(1), 13-18

[11] Pivovarenko Y. (2017) The Electric Potential of the Tissue Liquids of Living Organisms as a Possible Epigenetic Factor. Chemical and Biomolecular Engineering, 2(3), 159-164

[12] Spangenberg J. E. and Vennemann T. W. (2008) The stable hydrogen and oxygen isotope variation of water stored in polyethylene terephthalate (PET) bottles. Rapid Commun. Mass Spectrom, 22, 672-676

[13] Shevchenko V. B., Datsenko A. I., Shablykin O. V., Osadchuk T. V., Lyakhov A M., Pivovarenko Yu. V. and Makara V. A. (2012) Determination of ROS in the presence of biologically active substances by fluorescence of porous silicon. Ukrainian Biochemical Journal, 84(4), 67-71. In Russian

[14] Saenger V. (1987) Principles of structural organization of nucleic acids. Moscow: Mir. In Russian

[15] Pivovarenko, Y. (2017) An alternative strategy in cancer chemotherapy, aimed not at killing cancer cells, but the recovery of their DNA, modified by active oxygen // Biomedical Sciences, 3(5), 94-98

[16] Shpolsky, E. V. (1974) Atomic Physics, 1. Moscow: Nauka. In Russian.

[17] Tsu R., Bablic D. and Ioriatti L. Jr. (1997) Simple model for the dielectric constant of nanoscale silicon particle. J. Appl. Phys, 82(3), 1327-1329. 